\title{
COMPOSITION DU COMITÉ DE PROSPECTIVE
EN ENERGIE
}

Présidents

Sébastien CANDEL

Membre de l'Académie des sciences Membre de l'Académie des technologies Professeur à l'École centrale Paris

Bernard TISSOT

Membre de l'Académie des sciences Membre de l'Académie des technologies Président honoraire de la Commission nationale d'évaluation des études et recherches sur la gestion des matières et déchets nucléaires $\left(\mathrm{CNE}^{2}\right)$

\section{Membres}

Jean-Claude ANDRÉ

Roger BALIAN

Yves BRÉCHET

Membre de l'Académie des sciences Professeur émérite à l'université Pierre-etMarie-Curie et professeur honoraire à l'École polytechnique

Correspondant de l'Académie des sciencesMembre de l'Académie des technologies Directeur honoraire du Centre européen de recherche et de formation avancée en calcul scientifique (Cerfacs)

Membre de l'Académie des sciences Physicien

Membre de l'Académie des sciences Haut-commissaire à l'énergie atomique et aux énergies alternatives - Professeur à I'Institut polytechnique de Grenoble (INPG) et au laboratoire Science et ingénierie des matériaux et des procédés de Grenoble (SIMaP)

\section{Édouard BRÉZIN}


Catherine CÉSARSKY

Michel COMBARNOUS

Vincent COURTILLOT

Jean-Claude DUPLESSY

Pierre ENCRENAZ

Marc FONTECAVE

Robert GUILLAUMONT

Claude HENRY

Pierre JOLIOT

Guy LAVAL
Membre de l'Académie des sciences - Haut conseiller scientifique au Commissariat à l'énergie atomique et aux énergies alternatives (CEA)

Correspondant del'Académie des sciencesMembre de l'Académie des technologies Professeur émérite à l'université de Bordeaux 1

Membre de l'Académie des sciences Professeur à l'université Paris Diderot (Paris 7)

Membre de l'Académie des sciences Directeur de recherche émérite au Centre national de la recherche scientifique (CNRS)

Membre de l'Académie des sciences Professeur à l'université Pierre-et-MarieCurie

Membre de l'Académie des sciences Professeur au Collège de France

Membre de l'Académie des sciences Membre de l'Académie des technologies Professeur honoraire à l'université Paris-Sud Orsay

Professeur à l'IDDRI Sciences-Po Paris et à Columbia University

Membre de l'Académie des sciences Professeur honoraire au Collège de France

Membre de l'Académie des sciences Directeur de recherche émérite au Centre national de la recherche scientifique (CNRS) 
Marc PÉLEGRIN

Olivier PIRONNEAU

Michel POUCHARD

Paul-Henri REBUT

Didier ROUX

Jean SALENÇON

Jean-Marie TARASCON
Correspondant del'Académie des sciencesMembre de l'Académie de l'air et de l'espace - Membre de l'Académie des technologies - Haut conseiller honoraire à l'Office national d'études et de recherches aérospatiales (Onera)

Membre de l'Académie des sciences Professeur à l'université Pierre-et-MarieCurie

Membre de l'Académie des sciences Membre de l'Académie des technologies Professeur émérite à l'université de Bordeaux 1

Correspondant de l'Académie des sciencesConseiller scientifique au Commissariat à l'énergie atomique et aux énergies alternatives (CEA)

Membre de l'Académie des sciences Membre de l'Académie des technologies Directeur de la recherche et de l'innovation de Saint-Gobain

Membre de l'Académie des sciences Membre de l'Académie des technologies Ingénieur général honoraire des ponts et chaussées, professeur honoraire à l'École polytechnique et à l'École nationale des ponts et chaussées

Membre de l'Académie des sciences Professeur à l'université de Picardie Jules Verne et au Collège de France

\section{Ont également participé à la rédaction du rapport}

Yves BAMBERGER
Conseiller scientifique du Président d'EDF Membre de l'Académie des technologies 
Daniel CLÉMENT

Jean JACQUINOT

Claude JAUPART

François MOISAN

Xavier MONTAGNE

Satish SINGH
Directeur scientifique adjoint de l'Ademe

Conseiller auprès du Haut-commissaire à l'énergie atomique et aux énergies alternatives (CEA)

Membre de l'Académie des sciences Professeur à l'université Paris Diderot

Directeur de la stratégie et directeur scientifique de l'Ademe

Directeur adjoint à la Direction scientifique de l'IFP Énergies nouvelles (IFPEN)

Professeur à l'Institut de physique du globe de Paris - Laboratoire de géosciences marines -

Le chapitre 7 s'appuie sur une étude réalisée par l'Académie de l'air et de l'espace

\section{Liste des personnes auditionnées}

Jacques AMOUROUX

Olivier APPERT

Nicolas ARNAUD

Françoise BARBIER

Noël CAMARCAT

Jean-Michel DELBECQ
Professeur à l'université Pierre-et-MarieCurie

Président de l'IFPEN - Président de l'Ancre

Directeur de recherche - OSU-OREME CNRS

Directrice R\&D - Air Liquide

Délégué R\&D nucléaires et affaires internationales - EDF

Directeur scientifique R\&D nucléaire - EDF 
Françoise ELBAZ-POULICHET

Gilles FLAMANT

Jean-Claude GRENIER

Jean JACQUINOT

François KALAYDJIAN

Daniel KAPLAN

Alain LEUDET

Didier ROUX

Michel SÉRANNE

lonel SOLOMON
Directrice de recherche - OSU-OREME CNRS

Directeur de recherche - CNRS

Directeur de recherche - CNRS

Conseiller auprès du Haut-commissaire à l'énergie atomique et aux énergies alternatives (CEA)

Directeur de la Direction des technologies de développement durable - IFP Énergies nouvelles

Membre de l'Académie des sciences Membre de l'Académie des technologies Directeur de la société Fastlite

Conseiller scientifique au Commissariat à l'énergie atomique et aux énergies alternatives (CEA)

Membre de l'Académie des sciences Membre de l'Académie des technologies Directeur de la recherche et de l'innovation de Saint-Gobain

Chargé de recherche - OSU-OREME CNRS

Membre de l'Académie des sciences Membre de l'Académie des technologies Directeur de recherche émérite au CNRS

\section{Coordination éditoriale}

Joëlle FANON

Adjointe du directeur du service des publications de l'Académie des sciences 
